studies of the amyloid A4 protein precursor (APP) gene. Although this is also located on chromosome 21 , it does not contain the primary genetic defect in familial AD. However, the abnormal deposition of this protein is clearly a prominent feature of the neuropathology of AD. Studies of the several different forms of mRNA coded for by this gene and its regulation are described in a number of chapters. This work is likely to lead to an increased understanding of the pathogenesis of AD. However, a major unresolved issue remains that of how early in the chain of pathogenic events amyloid deposition is located.

Overall this book is of a higher standard than is usually encountered in conference proceedings and is exceedingly well presented. It will be of value to those working in this area but most of the chapters are probably too technical for those less well acquainted with the subject.

M J OWEN

\section{Practical Genetic Counselling}

3rd edition. By Peter S Harper. (Pp 306; £25.00.) Wright. 1988.

It is good to see the third edition of this book, which is unique in its comprehensive cover of most of the genetic problems encountered in clinical practice. It contains major changes and additions. There is a new chapter on congenital malformation syndromes and dysmorphology, while the rapidly advancing area of molecular genetics is covered in a much revised chapter.

The book is organised in three parts. Part 1 covers general aspects of genetic counselling in its broadest sense. The basics of mendelian, chromosomal, and molecular genetics are well described, as are the applications to carrier detection, prenatal diagnosis, risk determination, and segregation analysis. The final chapter in this section is devoted to 'The genetic counselling clinic' and reflects the author's great experience and practical approach and will be very valuable to those who are interested in setting up or understanding clinics of this sort.

Part 2 deals systematically with specific organ systems and their inherited disorders and has excellent references and sources of further information.

Part 3 deals with 'Genetic counselling and society'. There is much valuable information in an appendix on sources of information, including the addresses of genetic centres in Britain.

This book is probably not designed for clinical geneticists, although I suspect that many always have a copy near by. Its main attraction will be for perceptive clinicians in other specialties who realise how profoundly genetics is impinging on their practice. It is possible to dip into this book and find something about almost any genetic disorder, while the general chapters provide an excellent revision course for those whose clinical genetics is either very rusty or non-existent.

I suppose there is a danger that this book will encourage people to believe that they can cope with any genetic problem, but as the general awareness of the importance of genetics increases, most clinicians will appreciate that there are many pitfalls, not least of which are the problems created by genetic heterogeneity and the need for precise diagnosis of rare disorders.

The style and presentation of the book are very attractive although even this paragon among books contains occasional errors. However, one has to search hard for even minor mistakes, for example, figure 5.5 (a) is not a fully informative pedigree.

This book is without doubt one of the most useful currently available. It can be thoroughly recommended to any clinician or scientist interested in this rapidly advancing field.

RODNEY HARRIS

\section{Advances in Human Genetics}

Edited by Harry Harris and Kurt Hirschhorn. Volume 18. (Pp 383; \$59.50.) New York: Plenum Press. 1989.

As in previous volumes in this series, five review articles are presented, one largely clinical, one largely cytogenetic, and three which straddle the interface of molecular biology and clinical medicine.

In a clear and concise update of HLA disease association Bell, Todd, and McDevitt describe the structure and function of the major histocompatibility complex (MHC). The chapter concentrates on the class II region, with which the strongest disease associations have been found, associations which are being refined through sequencing of the class II loci. Although other factors, both genetic and environmental, play a role in the genesis of the diseases showing HLA association, unravelling the molecular basis of the associations will provide insight into a key step in their aetiology. Furthermore, the hope expressed by the authors, that peptide-MHC interactions underlying these diseases might in the future be modified directly, does not seem too remote.

Cohen and Levy review the major chromosome 
instability syndromes (in which they include xeroderma pigmentosum), detailing the spontaneous and induceable cytogenetic characteristics of each. The multiple complementation groups observed in xeroderma pigmentosum and ataxia telangiectasia are discussed together with the elucidation to date of the defects underlying these conditions, Bloom's syndrome and Fanconi's anaemia. Parts of the chapter are hard going for the non-specialist, particularly a section describing the conflicting evidence for circulating clastogens. The clinical features of these four conditions are briefly reviewed together, and a short section on prenatal diagnosis is provided. Results from the most recent molecular studies are to be found in the addenda in the back of the volume. I would have found most useful a flowchart summarising the distinguishing cytogenetic characteristics of these conditions and the related, but undiscussed, disorders.

In the third chapter, Robinson succinctly discusses the diverse spectrum of inherited metabolic disorders leading to lacticacidaemia. Attention is focused on recent advances in understanding of the structure, function, and dysfunction of the pyruvate dehydrogenase complex, components of the mitochondrial respiratory chain and pyruvate carboxylase. The genetics of the enzyme complexes involved in these central metabolic pathways is complex, but integration of molecular and biochemical research is helping to improve definition of this group of disorders, each of which is associated with great clinical heterogeneity.

The final chapters share a common theme of growth; in one Cohen gives a clinical review of overgrowth and the overgrowth syndromes, the other from Phillips and Vnencak-Jones is a clearly written summary of the genetics of growth hormone and its disorders. Cohen's review is extensively referenced and descriptive rather than mechanistic. It is aimed primarily at the clinician. It is easy to read and will prove interesting and useful to a variety of specialists. In the last review the anatomy, function, and regulation of the growth hormone gene cluster are covered. Molecular pathology is related to the recognised deficiency states. Although the chapter is brief and interesting the reader is perhaps reminded that it possesses the second of these virtues a little too often.
The fact that these reviews are all relevant to the clinical geneticist indicates the strength of the links between basic research and clinical practice in this discipline. The volume will be of interest and value to clinical and human geneticists alike.

J SAMPSON

Twinning and Twins

Edited by I MacGillivray, D M Campbell, and B Thompson. (Pp 321; £52.00.) Chichester: John Wiley. 1988.

The general public as well as the medical and scientific community are fascinated by twins. A number of eminent medical geneticists are either a twin themselves or have children who are twins. One might speculate on which is 'chicken and egg'.

Although this is a multi-author book with a number of contributors, many of the chapters are written by or written in conjunction with one of the three editors because of the long standing interest in twinning and twins in Aberdeen by the editors and their colleagues.

This book could be of interest to obstetricians and paediatricians as well as geneticists as it has wide variety of chapters covering the epidemiology; aetiology, and types of twins, physiological changes in and management of pregnancy, labour, and delivery, as well as the outcome as far as birth weight, intelligence, and congenital anomalies. The text is well referenced in general and the discussion on the possible mechanisms for congenital anomalies in twins was of particular interest.

A seemingly unavoidable criticism in multi-author texts is that the early chapters on aetiology, epidemiology, and factors affecting and types of twinning are, perhaps unavoidably, repetitive in the material they discuss and cite.

While this book fairly comprehensively covers most aspects of twinning and twins, it is to be recommended as a useful reference on the subject rather than a 'cover to cover' read.

R F Mueller 